\title{
Glutaryl-CoA Dehydrogenase Deficiency in Spain: Evidence of Two Groups of Patients, Genetically, and Biochemically Distinct
}

\author{
CHRISTIANE BUSQUETS, BEGOÑA MERINERO, ERNST CHRISTENSEN, JOSEP LLUÍS GELPÍ, \\ JAUME CAMPISTOL, MERCÈ PINEDA, EMILIO FERNÁNDEZ-ALVAREZ, JOSÉ M PRATS, \\ ANA SANS, ROSA ARTEAGA, MILAGROS MARTÍ, JAIME CAMPOS, \\ MERCEDES MARTÍNEZ-PARDO, ANTONIO MARTÍNEZ-BERMEJO, M LUISA RUIZ-FALCÓ, \\ JULIÁN VAQUERIZO, MODESTO OROZCO, MAGDALENA UGARTE, M JOSEP COLL, AND \\ ANTONIA RIBES
}

Institut de Bioquímica Clínica, Barcelona 08280, Spain [C.B., M.J.C., A.R.]; Centro de Diagnóstico de Enfermedades Moleculares, Universidad Autónoma, Madrid 28049, Spain [B.M., M.U.]; Department of Clinical Genetics, Rigshospitalet, Copenhagen 2100, Denmark [E.C.]; Departament de Bioquímica, Universitat de Barcelona, Barcelona 08028, Spain [J.L.G., M.O.]; Hospital Universitari Sant Joan de Déu, Esplugues de Llobregat 08950, Spain [M.P., E.F.-A., A.S.]; Hospital de Cruces, Baracaldo 48903, Spain [J.M.P.]; Hospital Marqués de Valdecilla, Santander 39008, Spain [R.A.]; Hospital Materno-Infantil de Las Palmas, Las Palmas 35016, Spain [M.M.]; Hospital Clínico, Madrid 28040, Spain [J.C.]; Hospital Ramón y Cajal, Madrid 28034, Spain [M.M.-P.]; Hospital Infantil La Paz, Madrid 28046, Spain [A.M.-B.]; Hospital Niño Jesús, Madrid 28009, Spain [M.L.R.-F.], Hospital Universitario Infanta Cristina, Badajoz 06010, Spain [J.V.]

\section{ABSTRACT}

Glutaryl-CoA dehydrogenase (GCDH) deficiency causes glutaric aciduria type I (GA I), an inborn error of metabolism that is characterized clinically by dystonia and dyskinesia and pathologically by neural degeneration of the caudate and putamen. Studies of metabolite excretion allowed us to categorize 43 GA I Spanish patients into two groups: group 1 (26 patients), those presenting with high excretion of both glutarate and 3-hydroxyglutarate, and group 2 (17 patients), those who might not be detected by routine urine organic acid analysis because glutarate might be normal and 3-hydroxyglutarate only slightly higher than controls. Single-strand conformation polymorphism (SSCP) screening and sequence analysis of the 11 exons and the corresponding intron boundaries of the GCDH gene allowed us to identify 13 novel and 10 previously described mutations. The most frequent mutations in group 1 were A293T and R402W with an allele frequency of $30 \%$ and $28 \%$, respectively. These two mutations were also found in group 2, but always in heterozygosity, in particular in combination with mutations V400M or R227P. Interestingly, mutations V400M and R227P were only found in group 2, and at least one of these mutations was found in 11 of 15 unrelated alleles, accounting together for 53\% of the mutant alleles in group 2. Therefore, it seems clear that two genetically and biochemically distinct groups of patients exist. The severity of the clinical phenotype seems to be closely linked to the development of encephalopathic crises rather than to residual enzyme activity or genotype. Comparison of GCDH protein with other acyl-CoA dehydrogenases (whose x-ray crystal structure has been determined) reveals that most of the mutations identified in GCDH protein seem to affect folding and tetramerization, as has been described for a number of mutations affecting mitochondrial $\beta$-oxidation acyl-CoA dehydrogenases. (Pediatr Res 48: 315-322, 2000)
GA I, glutaric aciduria type I
GCDH, glutaryl-CoA dehydrogenase
FAD, flavin adenine dinucleotide
ETF, electron-transfer flavoprotein
GC-MS, gas chromatography-mass spectrometry
MCAD, medium-chain acyl-CoA dehydrogenase
SCAD, short-chain acyl-CoA dehydrogenase
VLCAD, very-long-chain acyl-CoA dehydrogenase
IVD, isovaleryl-CoA dehydrogenase
SSCP, single-strand conformation polymorphism

Abbreviations
GA I (MIM 231670) is an autosomal recessive disorder caused by deficient or nonfunctional GCDH (EC 1.3.99.7). The

Received November 11, 1999; accepted March 1, 2000

Correspondence: Antonia Ribes, Institut de Bioquímica Clínica, C/Mejía Lequerica s/n, Edifici Helios III, Barcelona E-08028, Spain.

This work was supported by Fondo de Investigaciones Sanitarias (FIS) grant 97/0049-01. metabolic block leads to the accumulation of glutaric and 3-hydroxyglutaric acids as well as glutarylcarnitine in body fluids. The clinical picture is characterized by the sudden onset of a severe dystonic-dyskinetic disorder, hypotonia, irritability, macrocephaly, and degeneration of the caudate and putamen, which generally appear between 5 and 14 mo of age, but mild 
symptoms such as motor delay and hypotonia can be observed at earlier ages (1-4). However, clinical variability is common and some patients remain asymptomatic $(2,4)$. The condition is not exceedingly rare, and a frequency of 1:40,000 in Caucasians has been proposed (2).

The GCDH enzyme functions in the mitochondrial matrix as a homotetramer. The monomer is made up of 438 amino acids $(48.2 \mathrm{kD})$, of which the $44 \mathrm{~N}$-terminal residues are cleaved after being imported into the mitochondria. It also contains one molecule of FAD, which is reduced by ETF (5).

The human GCDH gene has been mapped to chromosome 19p13.2 (6), spans about $7 \mathrm{~Kb}$, and comprises 11 exons and 10 introns. The genomic structure and most of the intronic sequences have been published $(5,7,8)$ and the amino acid sequences of mouse, human, and pig GCDH are highly conserved (9). Single prevalent mutations, IVS1 $+5 \mathrm{G}>\mathrm{T}$ and A $421 \mathrm{~V}$, have been found in the Ojibway Indians of Canada (10) and in the Amish of Pennsylvania (7), respectively. However, mutations of the GCDH gene in general population are heterogeneous. To date, 66 disease-causing mutations and 6 polymorphisms and neutral mutations have been identified in the GCDH gene $(7,8,10-16)$, the most common mutation being R402W with an allele frequency of 12 to $16 \%(7,8)$.

In this study, we have analyzed metabolite excretion, GCDH activity, and the mutations responsible for GCDH deficiency in 43 Spanish patients with GA I. Our studies showed that there were two genetically and biochemically distinct groups of patients. We have attempted to establish a correlation between the GA I genotype and the clinical or biochemical phenotype.

\section{MATERIALS AND METHODS}

Patients. We studied 43 patients (35 unrelated families) with GA I from different areas of Spain. Diagnosis was established by clinical symptoms, characteristic findings on neuroimaging, and quantitative analysis of organic acids in urine. The disease was confirmed by analysis of GCDH activity in cultured skin fibroblasts, as previously described (17). Fibroblasts from nine patients were not available, but in six of them GCDH activity could be predicted on the basis of other patients belonging to the same GCDH genotype. Only in four families were the parents known to be consanguineous. Case histories of patients $4,13,19,22,23,25,26,27,28,34,38,39$, and 42 have been previously described (18-23).

In 24 patients the disease began abruptly, during infancy, with focal seizures or generalized convulsions, vomiting, and obtundation or lethargy, usually after an acute infectious illness. In 18 patients the onset was "insidious," with slowly developing psychomotor delay, hypotonia as dystonic postures, lethargy, or macrocephaly. The phenotype was classified according to the following criteria: "severe," when the patient showed dystonia (either static or progressive) and was unable to stand up, walk, or speak (25 patients); "mild," when dystonia was static or improving and the child was able to walk without help (11 patients); and "slight," when there were only minimal neurologic signs, and the child was able to lead a normal life ( 5 patients). Two patients were asymptomatic. The siblings of patients presented the same phenotype and clinical onset as their corresponding index case, except patient 11, who is an elder asymptomatic brother of patient 10 , and patient 17 , who is a younger brother of patient 16 with a milder clinical phenotype (Table 1).

The ethics committees of the participating hospitals and organizations approved the collection of samples on which these studies were performed.

Quantitative analysis of glutarate and 3-hydroxyglutarate. Organic acids were isolated by strong anion exchange (SAX) column extraction as described (24) and were analyzed by GC-MS (Hewlett-Packard 5890, Palo Alto, CA, U.S.A.) as trimethylsilyl (TMS)-derivatives. For quantitation, the mass spectrometry was operated in the single ion monitoring mode, and the following ion currents were measured: glutarate $\mathrm{m} / \mathrm{z}$ 261, 3-hydroxyglutarate $\mathrm{m} / \mathrm{z} 259$, and undecanedioate (internal standard) $\mathrm{m} / \mathrm{z}$ 345. The sodium salts of glutaric and undecanedioc acids were obtained from Sigma Chemical Co. (St. Louis, MO, U.S.A.) and 3-hydroxyglutaric acid was from Dr. Brunet (Organic Chemistry Department, Universidad Autónoma de Madrid, Spain).

Mutation analysis. Genomic DNA was prepared from fresh blood or from cultured skin fibroblasts by standard methods. Total RNA was prepared from cultured fibroblasts by the Ultraspec $^{\mathrm{TM}}$ RNA Isolation System (Biotecx, Laboratories, Houston, TX, U.S.A.).

Mutation screening of genomic DNA was performed by PCR amplification and SSCP analysis. PCR conditions and primers used were as described elsewhere $(7,14)$.

SSCP was performed at room temperature or at $4^{\circ} \mathrm{C}$. DNA fragments were detected after silver staining. The bands with mobility shifts were excised from the gel, purified (QIAquick Gel Extraction Kit; QIAGEN, GmbH Germany, Hilden, Germany) and reamplified, followed by sequencing using a Sequenase Version 2.0 Kit (USB, Amersham Pharmacia Biotech, Buckinghamshire, U.K.) and ${ }^{35}$ S-dATP. In some cases, PCR products were purified (QIAquick PCR Purification Kit; QIAGEN) and sequenced with the DNA sequencing kit, dye terminator cycle sequencing ready reaction (Perkin Elmer Applied Biosystems Division, Foster City, CA, U.S.A.).

For cDNA analysis, about $5 \mu \mathrm{g}$ of total RNA was transcribed into the first strand of cDNA in a $25-\mu \mathrm{L}$ reaction volume containing avian myeloblastosis virus (AMV) $1 \times$ buffer, $400 \mu \mathrm{M}$ dinucleotide triphosphate (dNTP), $10 \mathrm{U}$ AMV reverse transcriptase (Promega, Madison, WI, U.S.A.), $33 \mathrm{U}$ RNA in ribonuclease inhibitor (Promega), and $400 \mathrm{nM}$ of primer for $45 \mathrm{~min}$ at $52^{\circ} \mathrm{C}$. PCR amplification of cDNA was carried out using primers described elsewhere (10). PCR products were studied by SSCP analysis and sequenced as described above.

When possible, sequence changes were confirmed by restriction enzyme analysis as summarized in Table 2. To detect mutation A293T, a mismatch PCR was designed. We introduced an $\mathrm{HgaI}$ restriction site into the mutant PCR product. The two primers used were located on exon 8. The forward mismatch primer was F5' $^{\prime}$-CCTTCGGCTGCCTGAACGAC$3^{\prime}$, where the mismatch nucleotide is underlined, and the reverse primer was as described (7). 
Table 1. Genotype-phenotype correlation in 35 unrelated Spanish families with GA I

\begin{tabular}{|c|c|c|c|c|c|c|}
\hline \multirow[b]{2}{*}{ Patients } & \multirow[b]{2}{*}{ Genotype } & \multicolumn{2}{|c|}{ Metabolite excretion* } & \multirow{2}{*}{$\begin{array}{c}\text { \%GCD activity } \\
\text { in fibroblasts }\end{array}$} & \multirow[b]{2}{*}{ Clinical onset } & \multirow[b]{2}{*}{ Phenotype } \\
\hline & & Glutarate & 3-OH-Glutarate & & & \\
\hline \multicolumn{7}{|l|}{ Group 1} \\
\hline 3 & A293T /A293T & 1773 & 340 & $<0.5$ & Acute encephalopathy & Severe \\
\hline $4,5 \dagger$ & A293T /A293T & $4135 / 2166$ & $752 / 420$ & $<0.5$ & Insidious & Mild \\
\hline $6,7 \dagger$ & A293T /A293T & $500 / 3200$ & $461 / 843$ & n.d. & Insidious & Slight \\
\hline 8 & A293T/E90K & 1098 & 148 & 6 & Acute encephalopathy & Severe \\
\hline 10,11 & A293T /R402W & $772 / 2083$ & $264 / 262$ & n.d. & Acute encephalopathy/none & Severe/asymptomatic \\
\hline 12 & $\mathrm{~A} 293 \mathrm{~T} / \mathrm{R} 402 \mathrm{~W}$ & 8188 & 3573 & n.d. & Insidious & Severe \\
\hline 13 & $\mathrm{R} 402 \mathrm{~W} / \mathrm{R} 402 \mathrm{~W}$ & 1288 & 75 & $<0.5$ & Insidious & Severe \\
\hline 14 & $\mathrm{R} 402 \mathrm{~W} / \mathrm{R} 402 \mathrm{~W}$ & 2625 & 262 & n.d. & Insidious & Mild \\
\hline $15 t$ & $\mathrm{R} 402 \mathrm{~W} / \mathrm{R} 402 \mathrm{~W}$ & n.d. & n.d. & n.d. & Acute encephalopathy & Severe \\
\hline 16,17 & R402W /R128X & $4885 / 6644$ & $713 / 603$ & $<0.5$ & Acute encephalopathy & Severe/mild \\
\hline 23 & $\begin{array}{l}\text { 423-424delGC } \\
\text { /R383C }\end{array}$ & 7932 & 1074 & $<0.5$ & Insidious & Asymptomatic \\
\hline 24 & A304T/ L309W & 1673 & 795 & $<0.5$ & Insidious & Slight \\
\hline $25,26 \dagger$ & $\mathrm{A} 316 \mathrm{D} / \mathrm{A} 316 \mathrm{D}$ & $6164 / 3556$ & $626 / 420$ & $<0.5$ & Acute encephalopathy & Severe \\
\hline \multicolumn{7}{|l|}{ Group 2} \\
\hline 27 & V400M /V400M & 2 & 18 & 10 & Acute encephalopathy & Severe \\
\hline 28 & $\mathrm{~V} 400 \mathrm{M} / \mathrm{V} 400 \mathrm{M}$ & 2 & 185 & 5 & Acute encephalopathy & Severe \\
\hline 29 & V400M /V400M & 5 & 114 & n.d. & Acute encephalopathy & Severe \\
\hline 30 & $\mathrm{~V} 400 \mathrm{M} / \mathrm{R} 402 \mathrm{~W}$ & 25 & 571 & 1 & Acute encephalopathy & Severe \\
\hline 31 & $\mathrm{~V} 400 \mathrm{M} / \mathrm{R} 402 \mathrm{~W}$ & 12 & 175 & 2 & Acute encephalopathy & Mild \\
\hline 32 & V400M /R402W & 69 & 429 & 3 & Acute encephalopathy & Severe \\
\hline 33 & V400M /unknown & 4 & 16 & 34 & Insidious & Severe \\
\hline
\end{tabular}

n.d., not done.

* Values are expressed in mmol/mol creatinine. Median and range for glutarate and 3-hydroxyglutarate in 42 independent controls were 3 (2-10) and 7 (2-14), respectively.

$\dagger$ Siblings with the same enzyme activity, clinical onset and phenotype than the corresponding index case.

$\$$ Patient 15 died and no samples were available; the presumptive diagnosis of GA I was established due to clinical features and demonstration of heterozygosity in the parents.

Mutations were named according to suggested nomenclature (25). Throughout this article, all mutations except frameshift are referred to by their amino acid-based name.

\section{RESULTS}

Biochemical and clinical findings. The use of quantitative, as opposed to qualitative, organic acid analysis allowed us to categorize the patients into two groups (Table 1): Group 1 (26 patients) comprised those who could be diagnosed simply by routine GC-MS organic acid analysis in urine. Patients presented with high excretion of both glutarate and 3-hydroxyglutarate, although the excretion of glutarate was always higher than that of 3-hydroxyglutarate. Group 2 (17 patients) comprised those who might go undetected by routine screening because glutarate excretion can be normal and that of 3-hydroxyglutarate might be only slightly increased. In contrast to group 1 patients, those in group 2 presented with a higher 3-hydroxyglutarate excretion than that of glutarate. The median and range $(\mathrm{mmol} / \mathrm{mol}$ creatinine) of glutarate and 3-hydroxyglutarate excretion in 42 control urine samples were $3(2-10)$ and $7(2-14)$, respectively; values for group 1 patients were 2817 (204-8955) and 420 (75-3573), respectively. In contrast, group 2 showed a median value for glutarate, $10(2-84)$, which is within the range of controls, whereas the median value for 3-hydroxyglutarate, 101 (16-571), fell within the range of group 1 . When possible, only those values of glutarate and 3-hydroxyglutarate obtained at the time of diagnosis were considered to obviate the possible influence of any treatment. 
Table 2. Summary of all mutations and their frequencies in 35 unrelated Spanish families with GA I

\begin{tabular}{|c|c|c|c|c|c|}
\hline \multirow[b]{2}{*}{ Location } & \multirow[b]{2}{*}{ Nucleotide change } & \multirow[b]{2}{*}{ Effect on protein } & \multirow[b]{2}{*}{ Restriction enzyme $\dagger$} & \multicolumn{2}{|c|}{ No. alleles } \\
\hline & & & & Group 1 & Group 2 \\
\hline \multicolumn{6}{|l|}{ Missense } \\
\hline Exon 3 & $304 \mathrm{G}>\mathrm{A} \dagger$ & E90K* & & 1 & 0 \\
\hline Exon 5 & $392 \mathrm{C}>\mathrm{T}^{\dagger}$ & S119L* & & 1 & 0 \\
\hline Exon 5 & $431 \mathrm{G}>\mathrm{A} \dagger$ & R132Q* & $\operatorname{AciI}(-)$ & 1 & 0 \\
\hline Exon 5 & $499 \mathrm{~T}>\mathrm{C}$ & $\mathrm{Y} 155 \mathrm{H}$ & $\operatorname{NcoI}(+)$ & 0 & 1 \\
\hline Exon 6 & $577 \mathrm{G}>\mathrm{A}$ & E181K* & & 2 & 0 \\
\hline Exon 6 & $619 \mathrm{G}>\mathrm{A}$ & A195T & $\operatorname{BanI}(-)$ & 1 & 0 \\
\hline Exon 7 & $716 \mathrm{G}>\mathrm{C}$ & R227P & $\operatorname{NlaIV}(+)$ & 0 & $4(13 \%)$ \\
\hline Exon 7 & $800 \mathrm{C}>\mathrm{T} \uparrow$ & S255L* & & 0 & 1 \\
\hline Exon 8 & $913 \mathrm{G}>\mathrm{A} \dagger$ & A293T & $\operatorname{HgaI}(-) \ddagger$ & $12(30 \%)$ & $2(7 \%)$ \\
\hline Exon 8 & $946 \mathrm{G}>\mathrm{A}$ & A304T* & $\operatorname{AluI}(-)$ & 1 & 0 \\
\hline Exon 10 & $1240 \mathrm{C}>\mathrm{T} \dagger$ & R402W & $\operatorname{MspI}(-)$ & $11(28 \%)$ & $3(10 \%)$ \\
\hline Exon 11 & $1334 \mathrm{C}>\mathrm{T} \dagger$ & $\mathrm{A} 433 \mathrm{~V}^{*}$ & $M w o I(+)$ & 0 & 2 \\
\hline \multicolumn{6}{|l|}{ Deletions } \\
\hline Exon 5 & 423-424 del GC & Truncated on $185 \mathrm{aa}^{*}$ & $\operatorname{Alu} \mathrm{I}(-)$ & 1 & 0 \\
\hline Exon 7 & $884 \mathrm{del} \mathrm{T}$ & Truncated on 289 aa* & $\operatorname{MspI}(+)$ & 0 & 1 \\
\hline Exon 10 & $1209 \mathrm{del} \mathrm{G}$ & Truncated on 399 aa & & 3 & 1 \\
\hline \multicolumn{6}{|l|}{ Nonsense } \\
\hline Exon 5 & $418 \mathrm{C}>\mathrm{T} \dagger$ & $\mathrm{R} 128 \mathrm{X}^{*}$ & $\operatorname{Sau} 961(-)$ & 1 & 0 \\
\hline
\end{tabular}

The shaded region highlights mutations that appear in both groups.

* New mutations found in this study.

$\uparrow$ Substitutions found at hypermutable $\mathrm{CpG}$ sites; $(+)$ mutation creates the restriction site, $(-)$ mutation destroys the site.

$\ddagger$ Restriction site created by a mismatch primer (see Materials and Methods).

Glutarylcarnitine in blood spots was tested in six patients from group 2: patient 38 (Dr. Millington, Duke University, Durham, NC, U.S.A.) and patients 30, 34, 36, 37, and 41 (Dr. Hoffmann, Philipps-Universitat, Marburg, Germany). All of them gave negative results (even under carnitine supplementation). This indicates that patients with this metabolite excretion pattern would not be detected in neonatal screening programs employing tandem mass spectrometry.

In group 1, all but two patients had undetectable GCDH activity in fibroblasts ( $<0.5 \%$ of controls), whereas in group 2 , the residual activities were usually higher (Table 1).

Acute encephalopathy marked the clinical onset in 24 patients, of whom 21 presented the severe phenotype. Insidious onset was found in 18 patients, of whom only 4 presented the severe phenotype (Table 1). Interestingly, $71 \%$ of group 2 patients presented the severe phenotype, whereas it was only found in $50 \%$ of group 1 patients (Table 1). Therefore, the severity of the clinical phenotype does not seem to be directly related to the severity of the biochemical phenotype.

Mutation analysis. SSCP screening of the 11 exons and intron boundaries of the GCDH gene allowed us to identify 13 novel (10 missense, 2 deletions, and 1 nonsense) and 10 already known mutations, which account for 99\% (69/70) of the total number of mutant alleles (Table 2). Sequence analysis of GCDH cDNA and genomic DNA of patient 33 revealed that mutation V400M was the only consistent change in the entire protein-coding region, suggesting that the other mutations lie in the promoter region or elsewhere within the intronic sequences. Of the 23 mutations identified only three were common to both groups (A293T, R402W, and 1209delG). However, these mutations were found in group 2 always in heterozygosity, in particular in combination with mutations V400M or R227P. The latter mutations accounted for $53 \%$ of the group 2 alleles and were exclusive to this group, whereas R402W and A293T mutations, accounted for $58 \%$ of the group 1 alleles (Table 2). None of the missense mutations was found in 100 unrelated control chromosomes. Mendelian inheritance of the mutations was confirmed by use of a restrictiondigestion test, where available, or by SSCP pattern, in the DNA of the parents of 25 families.

Flow chart for the diagnosis of GA I. The flowchart depicted in Figure 1, based on the biochemical and mutational results reported here, is now being used in our laboratory. It is especially useful for the diagnosis of group 2 patients, and in most cases means that the measurement of GCDH activity is not necessary. Patients 29, 33, and 36 were, in fact, diagnosed using this flowchart.

\section{DISCUSSION}

The aim of this study was to identify the mutations responsible for GCDH deficiency in the Spanish patients to investigate a possible correlation between GA I genotypes and clinical and biochemical phenotypes. 


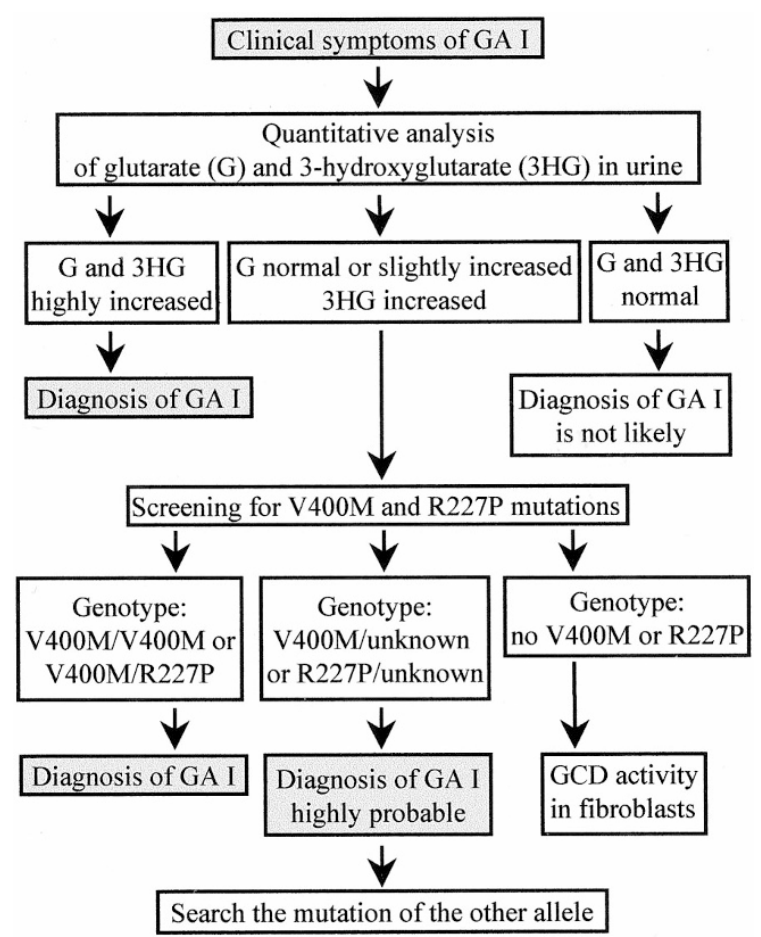

Figure 1. Flow-chart for the diagnosis of GA I.

Mutational spectrum. We report 13 novel mutations in the GCDH gene. This means that, at present, a total of 79 potentially pathogenic mutations are known. Missense mutations are more frequently detected than nonsense mutations or deletions, and here $55 \%$ of nucleotide substitutions were found at hypermutable CpG sites in the GCDH gene (Table 2). Deletions and nonsense mutations all result in truncation of the mutant protein before the $\alpha$-proton-abstracting base (E414) in enzyme catalysis (5); consequently, the encoded protein would not be expected to have any residual activity. Several lines of evidence suggest that missense mutations may be pathogenic: (1) after screening all of the coding region of the GCDH gene, no other mutations were found in the patients; (2) the nucleotide changes were not present in 100 normal chromosomes analyzed; and (3) all the amino acid residues, except R161 and A316, are conserved in pig and mouse GCDH homologs $(5,9)$.

According to the pattern of metabolite excretion, patients were categorized into two groups. In group 1, we have found 15 different mutations, of which 9 are novel. In contrast to other reports $(7,8,15)$, we found a particular prevalence not only for mutation R402W (28\%) but also for mutation A293T (30\%). In addition, we have recently found linkage disequilibrium between mutation $\mathrm{R} 402 \mathrm{~W}$ and a specific haplotype, suggesting a single origin for this mutation (26). In group 2, we have found 11 mutations, of which 4 are novel. The most striking result from this group was the strong association with mutations V400M and R227P, which together account for more than $50 \%$ of mutant alleles. We have previously shown that the R227P allele was associated with minimal glutarate excretion when heterozygous (13) and it has recently been confirmed in a patient with genotype E365K/R227P, whereas patients homozygous for mutation E365K present with large amounts of glutarate and 3-hydroxyglutarate in urine (27). The same applies when mutation A293T was found in homozygosity, whereas in heterozygosity with R227P the biochemical phenotype changed to that of group 2 (Table 1). Consequently, $\mathrm{R} 227 \mathrm{P}$ seems to be one of the causative mutations of this phenomena, but it is not clear how this mutation acts $(7,8)$. It might be the case that in these patients the decarboxylase activity was more severely affected than that of the dehydrogenase, but this was not sustained when mutation R227P was expressed in Escherichia coli, and partial reactions (dehydrogenation and decarboxylation) of GCDH activity were measured (28). A similar phenomena has been observed with mutation V400M. Genotypes V400M/R227P (patients 34, 35, and 36) and V400M/unknown (patient 33) presented the highest residual enzyme activities in fibroblasts, similar to those in the heterozygous range. If we take into account that patients homozygous for mutation V400M have lower enzyme activities than those compound heterozygous for V400M/R227P, a complementary effect between these two alleles might be speculated. Other private mutations (F71S, R161Q, and $\mathrm{S} 255 \mathrm{~L}$ ) were also found to be associated with this group of patients.

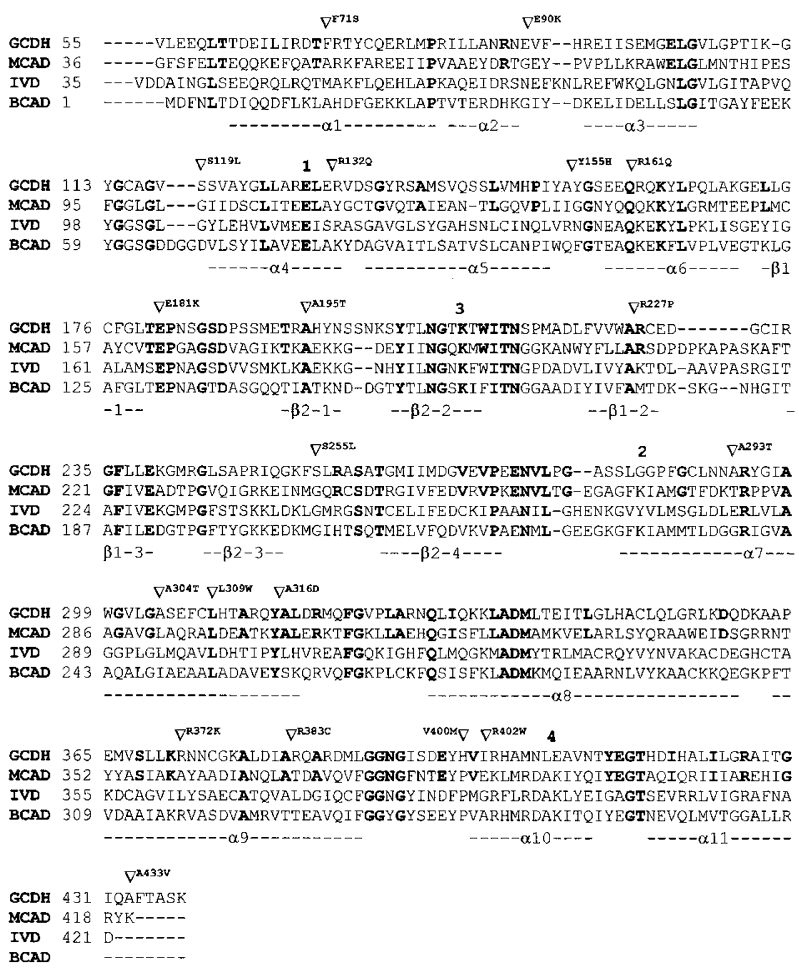

Figure 2. Alignment of the human GCDH sequence with the sequences of three acyl-CoA dehydrogenases (ACD) with known three-dimensional structure: pig MCAD (29), human IVD (30), and bacterial SCAD or BCAD (31). The GCDH sequence was aligned manually with the reference sequences taking into account conserved residues, residues in contact with substrates, and also the suitability of hydrophobicity and secondary structure profiles. All numbering is according to the precursor enzymes. The names of $\alpha$-helices and $\beta$-sheets are given below their sequence. The position of the amino acid change due to each missense mutation identified is shown above its sequence with downward-pointing arrows. Residues shared by all ACD or residues that are identical in GCDH and MCAD are highlighted. Positions marked by a number are described in detail in the text: 1, E-129 in GCDH; 2, G-284 in GCDH; 3, K-164 in mature MCAD (K189 in precursor MCAD); 4, E-408 in GCDH. 
Effects of GCDH mutations on protein structure. Human GCDH displays a high degree of homology with other acylCoA dehydrogenases (Fig. 2), therefore, comparison with the x-ray structure of MCAD (29), IVD (30) and SCAD or butyrylCoA dehydrogenase (BCAD) (31) may help to explain the molecular consequences of the identified mutations. As described for MCAD (29), the monomer structure of GCDH can be divided in three regions with a distinct secondary structure. Region A, from R-45 to E-172 (Fig. 2), consists of a bundle of six $\alpha$-helices that does not appear to be directly involved in either substrate binding or oligomerization (except for the first five residues, which might participate in oligomerization). In this region we found six mutations (F71S, E90K, S119L, $\mathrm{Y} 155 \mathrm{H}, \mathrm{R} 132 \mathrm{Q}$, and R161Q), most of which are associated with residual activity. Two of these, F71S and S119L, may affect protein stability because of a change in polarity, which is, moreover, maintained among reference structures. In particular, the change in polarity brought about by F71S could prevent the salt bridge formation between residues R72 and E129, impairing GCDH folding, as has been described for mutations R28C and R22W in MCAD and SCAD proteins, respectively $(32,33)$. Residue Y-155 forms a hydrogen bond with G-284, which lies near the adenine ring of CoA. Therefore, mutation $\mathrm{Y} 155 \mathrm{H}$ might compromise substrate binding. The consequences for mutations E90K, R132Q, and R161Q are less evident.

Region B, from L-173 to P-278 (Fig. 2), is a $\beta$-sandwich in close proximity to FAD. In this region we found four mutations, two of them (E181K and A195T) were associated with group 1 patients, whereas the other two (R227P and S255L) were found in group 2 patients. The substitution E181K may alter the local fold around the riboflavine moiety of FAD. Moreover, residue E-181 is homologous to residue E-137 in MCAD, the carboxyl of which is crucial for $\beta$-domain folding, and which has been described to affect tetramerization (34). A homologue disease-causing mutation, E178K, in VLCAD has also been identified (35). A similar effect could be predicted for mutation S255L. The mutation A195T leads to an increase in the volume and polarity of a conserved residue buried inside the $\beta$-sandwich, and this might alter the stability of the whole region. Mutation R227P lies in a region that might be involved in the ETF interaction site, as suggested for MCAD protein (36).

Region C consists of a bundle of four antiparallel $\alpha$-helices, the latter split in two halves. Loops connecting the helices are directly involved in the interaction with FAD molecule, the loop between $\alpha 7$ and $\alpha 8$ is in contact with the adenine ring, and the loop between $\alpha 9$ and $\alpha 10$ (including a patch of conserved glycine residues) is in contact with the pyrophospate group of the dinucleotide (Fig. 3). Moreover, FAD molecules interact at the same time with residues from the two subunits in such a way that FAD binding and subunit aggregation are closely linked. This region is structurally the most important, as regions $\mathrm{C}$ of the four subunits form the core of the tetramer. Mutations in this region are expected to compromise both FAD binding and intersubunit contacts. Figure 3 shows the position of the mutations found in this region (A293T, A304T, L309W, A316D, R372K, R383C, V400M, and R402W), together with

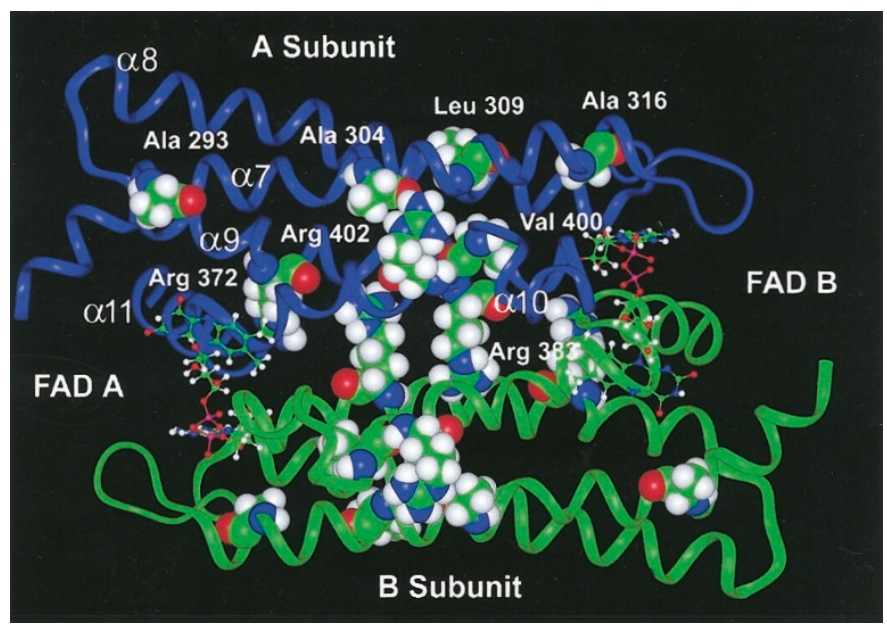

Figure 3. Location of GCDH mutations found in region C. Ribbon representation of region $\mathrm{C}$, comprising $\alpha 7$ to $\alpha 10-11$ (from 282 to 438 residues), of two subunits of the GCDH model. The view is oriented along the binary symmetry axis that relates the two subunits. Side chains of the mutated residues are shown as space-fill models and the two FAD molecules are indicated as ball and stick representations. Loops between $\alpha 7$ and $\alpha 8$ (adenine ring) and $\alpha 9$ and $\alpha 10$ (pyrophospate) interact directly with the coenzyme. All calculations were performed in a Silicon Graphics R8000 workstation using the molecular modeling programs Insight II, Homology, and Discover (Molecular Simulations, Inc., Princeton, NJ, U.S.A.).

a ribbon representation of regions $\mathrm{C}$ of two subunits. All of these mutations, except V400M, were associated with a lack of residual enzyme activity. The residual activity found in patients harboring mutation V400M can probably be accounted for by the fact that this does not constitute a drastic change. Three of these mutations (A293T, R402W, and V400M) were expressed in E. coli (15) and the results for R402W and V400M were inconsistent with the biochemical phenotype of our patients. The R $402 \mathrm{~W}$ mutant has been shown to have some residual activity (3\%), whereas the residual activity for mutant $\mathrm{V} 400 \mathrm{M}$ is almost undetectable $(0.8 \%)$; the contrary has been observed when GCDH was assayed in fibroblasts. These results reflect the difference between in vivo and in vitro analyses, as also observed in other diseases (37).

Genotype-phenotype correlation. Considerable clinical variability in patients with GA I has been reported (2-4). Patient 10 is an extreme example of this heterogeneity. This child presented a severe phenotype and died at $2 \mathrm{y}$ of age, whereas her elder GA I brother is asymptomatic at $4 \mathrm{y}$ of age (data not shown). Another apparent discrepancy of this disease is that a severe phenotype was recorded with greater frequency among group 2 patients (71\%), the biochemical phenotype of whom is less severe than that of group 1 . Therefore, it seems clear that high residual activity and low metabolite excretion do not prevent the development of a severe clinical phenotype (see patients 33 and 36). The severity of the phenotype seems to be closely linked to the development of encephalopathic crises, often in association with high fever. In our series, 91\% of patients with encephalopathic onset presented a severe phenotype, and prevention of the encephalopathic crises is the only treatment available to avoid further deterioration. Thus, environmental factors, such us exposure to metabolic stress, are probably one of the main determinants of the clinical 
phenotype, irrespective of the GA I genotype. This is by no means a new finding in the history of acyl-CoA dehydrogenase deficiencies; in MCAD deficiency, patients with the most severe mutations may remain asymptomatic for years and patients with the mildest MCAD mutations may present with the most severe symptoms (38). Similar observations have been made in SCAD deficiency (39).

It is interesting to note that, in contrast to glutarate, the concentration of 3-hydroxyglutarate in urine is similar in both groups of patients. This compound, at concentrations much lower than glutarate, has been reported to induce massive nerve cell lesions in vitro $(40,41)$. Most probably, 3-hydroxyglutarate arises from $\beta$-oxidation of the intramitochondrial increased glutaryl-CoA through the $\beta$-oxidation enzyme, MCAD (1), but this enzyme is not expressed in brain (42) and, consequently, is unable to produce 3-hydroxyglutarate in this tissue. It is thus difficult to link brain damage to this metabolite, but it might be the case that 3-hydroxyglutarate crosses the blood-brain barrier due to increased permeability during viral or bacterial infections (43-45) and perhaps then provokes brain lesions and encephalopathic crisis. The fact that 3-hydroxyglutarate did not increase in cerebrospinal fluid during clinical stability (A.R., personal observation) and that other authors found it increased during an acute illness (46) favor this hypothesis.

From this study we can conclude the following: (1) For certain diseases it is necessary to perform mutation analysis in every population. In earlier reports $(7,8,15)$ many private mutations were reported because, it would seem, the studies were conducted in heterogeneous populations. (2) Recently, it has been speculated that the number of group 2 patients was probably small (3), but this is not the case in our population, where $40 \%$ of patients were found to belong to this group. Whether this is a particular genetic characteristic of our population or reflects the results of ongoing attempts to improve diagnosis $(18,22,23,47)$ remains to be established. (3) The knowledge of GA I genotypes might be useful for prenatal diagnosis, particularly for families with high residual enzyme activity. (4) Comparison of GCDH protein with other crystalsolved acyl-CoA dehydrogenases reveals that most of the mutations identified might affect folding and tetramerization, as has been described for a number of mutations affecting mitochondrial $\beta$-oxidation acyl-CoA dehydrogenases. However, further experimental studies are needed to demonstrate this definitively. (5) With a few exceptions, a marked correlation between genotype and biochemical phenotype was found. However, no correlation was found between clinical symptoms, enzyme activities, and metabolite excretion in these Spanish GA I patients, which suggests that other factors contribute to the onset and severity of the disease.

Acknowledgments. The authors thank Dr. S.I. Goodman (University of Colorado School of Medicine, Denver, CO, U.S.A.), who provided us with information on GCDH gene structure so that we might undertake our molecular studies. We also thank C. Ogg and C. Llordés for their excellent technical assistance, and Laura Gort for her help and advice concerning mutation studies. We also thank the GA I families and the following pediatricians and biochemists who contributed to this study by providing fibroblasts or blood samples: J. Artigas, G. Pintos, P. Villanueva, M.A. Ruiz, M. Tallada, M.A. Vilaseca, M.A. Fernández, E. Riudor, M. Aparicio, G. Lorenzo, I. Corral, C. Garzo, G. Bueno, C. Pedrón, and J.C. Cabrera.

\section{REFERENCES}

1. Goodman SI, Frerman FE 1995 Organic acidemias due to defects in lysine oxidation: 2-ketoadipic acidemia and glutaric acidemia. In: Scriver CR, Beaudet AL, Sly WS, Valle D (eds) The Metabolic and Molecular Bases of Inherited Disease, 7th Ed. McGraw-Hill, New York, pp 1451-1460

2. Superti-Furga A, Hoffman G 1997 Glutaric aciduria type I (glutaryl-CoA dehydrogenase deficiency): advances and unanswered questions. Eur J Pediatr 156:821-828

3. Baric I, Zschocke J, Christensen E, Duran M, Goodman SI, Leonard JV, Müller E, Morton DH, Superti-Furga A, Hoffmann GF 1998 Diagnosis and management of glutaric aciduria type I. J Inherit Metab Dis 21:326-340

4. Hoffmann GF, Zschocke J 1999 Glutaric aciduria type I: from clinical, biochemical and molecular diversity to successful therapy. J Inherit Metab Dis 22:381-391

5. Goodman SI, Kratz LE, Di Giulio KA, Biery BJ, Goodman KE, Isaya G, Frerman FE 1995 Cloning of glutaryl-CoA dehydrogenase cDNA, and expression of wild type and mutant enzymes in Escherichia coli. Hum Mol Genet 4:1493-1498

6. Greenberg CR, Duncan AMV, Gregory CA, Singal R, Goodman SI 1994 Assignment of human glutaryl-CoA dehydrogenase gene (GCDH) to the short arm of chromosome 19 (19p13.2) by in situ hybridisation and somatic cell hybrid analysis. Genomics 21:289-290

7. Biery BJ, Stein DE, Morton DH, Goodman SI 1996 Gene structure and mutations of glutaryl-CoA dehydrogenase: impaired association of enzyme subunits that is due to an A421V substitution causes glutaric acidemia type I in the Amish. Am J Hum Genet 59:1006-1011

8. Schwartz M, Christensen E, Superti-Furga A, Brandt NJ 1998 The human glutarylCoA dehydrogenase gene: report of intronic sequence and of 13 novel mutations causing glutaric aciduria type I. Hum Genet 102:452-458

9. Koeller DM, DiGiulio KA, Angeloni SV, Dowler LL, Frerman FE, White RA, Goodman SI 1995 Cloning, structure, and chromosome localization of the mouse glutaryl-CoA dehydrogenase gene. Genomics 28:508-512

10. Greenberg CR, Reimer D, Singal R, Triggs-Raine B, Chudley AE, Dilling LA, Philipps S, Haworth JC, Seargeant LE, Goodman SI 1995 A G-to-T transversion at the +5 position of intron 1 in the glutaryl-CoA dehydrogenase gene is associated with the Island Lake variant of glutaric acidemia type I. Hum Mol Genet 4:493-495

11. Haworth JC, Singal R, Goodman SI, Greenberg CR 1994 TaqI polymorphism in intron 2 of the GCDH gene. Hum Mol Genet 3:678

12. Anikster Y, Shaag A, Joseph A, Mandel H, Ben-Zeev B, Christensen E, Elpeleg ON 1996 Glutaric aciduria type I in the Arab and Jewish communities in Israel. Am J Hum Genet 59:1012-1018

13. Christensen E, Ribes A, Busquets C, Pineda M, Duran M, Poll-The BT, Greenberg CR, Leffers H, Schwartz M 1997 Compound heterozygosity in the glutaryl-CoA dehydrogenase gene with R227P mutation in one allele is associated with no or very low free glutarate excretion. J Inherit Metab Dis 20:383-386

14. Busquets C, Coll MJ, Christensen E, Campistol J, Clusellas N, Vilaseca MA, Ribes A 1998 Feasibility of molecular prenatal diagnosis of glutaric aciduria type $\mathrm{I}$ in chorionic villi. J Inherit Metab Dis 21:243-246

15. Goodman SI, Stein DE, Schlesinger S, Christensen E, Schwartz M, Greenberg CR, Elpeleg ON 1998 Glutaryl-CoA dehydrogenase mutations in glutaric acidemia (type I): review and report of thirty novel mutations. Hum Mutat 12:141-144

16. Ikeda H, Kimura T, Ikegami T, Kato M, Matsunaga A, Yokoyama S, Yamaguchi S, Ohura T, Hayasaka K 1998 Novel mutations of the glutaryl-CoA dehydrogenase gene in two Japanese patients with glutaric aciduria type I. Am J Med Genet 80:327-329

17. Christensen E 1983 Improved assay of glutaryl-CoA dehydrogenase in cultured cells and liver: application to glutaric aciduria type I. Clin Chim Acta 129:91-97

18. Campistol J, Ribes A, Alvarez L, Christensen E, Millington DS 1992 Glutaric aciduria type I: unusual biochemical presentation. J Pediatr 121:83-86

19. Prats JM, Ribes A, Briones MP, Garaizar C, Sanjurjo P 1993 Aciduria glutarica tipo I. An Esp Pediatr 38:343-347

20. Martínez-Lage JF, Casas C, Fernandez MA, Puche A, Rodríguez-Costa T, Poza M 1994 Macrocephaly, dystonia, and bilateral temporal arachnoid cysts: glutaric aciduria type I. Childs Nerv Syst 10:198-203

21. Artigas J, Ribes A, Rovira A, Lorente I, Briones MP 1995 Aciduria glutárica tipo I con quistes aracnoideos. Rev Neurol 23:153-156

22. Merinero B, Pérez-Cerdá C, Font LM, Garcia MJ, Aparicio M, Lorenzo G, Martinez Pardo M, Garzo C, Martínez-Bermejo A, Pascual Castroviejo I, Christensen E, Ugarte M 1995 Variable clinical and biochemical presentation of seven Spanish cases with glutaryl-CoA dehydrogenase deficiency. Neuropediatrics 26:238-242

23. Pineda M, Ribes A, Busquets C, Vilaseca MA, Aracil A, Christensen E 1998 Glutaric aciduria type I with high residual glutaryl-CoA dehydrogenase activity. Dev Med Child Neurol 40:840-842

24. Verhaeghe BJ, Lefevere MF, De Leenheer AP 1988 Solid-phase extraction with strong anion-exchange columns for selective isolation and concentration of urinary organic acids. Clin Chem 3416:1077-1083

25. Antonorakis SE, and the Nomenclature Working Group 1998 Recommendations for a nomenclature system for human gene mutations. Hum Mutat 11:1-3

26. Busquets C, Coll MJ, Ribes A 1999 Evidence of a single origin for the most frequent mutation R402W causing glutaryl-CoA dehydrogenase deficiency. Identification of 3 
novel polymorphisms and haplotype definition. Hum Mutat, Mutation in Brief \#291 on-line.

27. Nyhan WL, Zschocke J, Hoffmann G, Stein DE, Bao L, Goodman SI 1999 GlutarylCoA dehydrogenase deficiency presenting as 3-hydroxyglutaric aciduria. Mol Genet Metab 66:199-204

28. Liesert M, Zschocke J, Hoffmann GF. Mühlhäuser N, Buckel W 1999 Biochemistry of glutaric aciduria type I: Activities of in vitro expressed wild-type and mutan cDNA encoding human glutaryl-CoA dehydrogenase. J Inherit Metab Dis 22:256258

29. Kim J-JP, Wang M, Paschke R 1993 Crystal structures of medium-chain acyl-CoA dehydrogenase from pig liver mitochondria with and without substrate. Proc Natl Acad Sci U S A 90:7523-7527

30. Tiffany KA, Roberts DL, Wang M, Paschke, Mohsen A-WA, Vockley, Kim J-JP 1997 Structure of human isovaleryl-CoA dehydrogenase at $2.6 \AA$ resolution: structural basis for substrate specificity. Biochemistry 36:8455-8464

31. Djordjevic S, Pace CP, Stankovich MT, Kim J-JP 1995 Three-dimensional structure of butyryl-CoA dehydrogenase from Megasphaera elsdenii. Biochemistry 34:21632171

32. Bross P, Jespersen C, Jensen TG, Andresen BS, Kristensen MJ, Winter V, Nandy A Kräutle F, Ghisla S, Bolund L, Kim J-JP, Gregersen N 1995 Effects of two mutations detected in medium chain acyl-CoA dehydrogenase (MCAD)-deficient patients on folding, oligomer assembly, and stability of MCAD enzyme. J Biol Chem 17:1028410290

33. Naito E, Indo Y, Tanaka K 1990 Identification of two variant short chain acyl-CoA dehydrogenase alleles, each containing a different point mutation in a patient with short chain acyl-CoA dehydrogenase deficiency. J Clin Invest 85:1575-1582

34. Saijo T, Kim J-JP, Kuroda Y, Tanaka K 1998 The roles of threonine-136 and glutamate-137 of human medium chain acyl-CoA dehydrogenase in FAD binding and peptide folding using site-directed mutagenesis: creation of an FAD-dependent mutant, T136. Arch Biochem Biophys 358:49-57

35. Andresen BS, Olpin S, Poorthuis BJ.H.M, Scholte JR, Vianey-Saban C, Wanders R, Ijlst L, Morris A, Pourfarzam M, Bartlett K, Baumgartner ER, deKlerk JBC, Schroeder LD, Corydon TJ, Lund H, Winter V, Bross P, Bolund L, Gregersen N 1999 Clear correlation of genotype with disease phenotype in very-long-chain acyl-CoA dehydrogenase deficiency. Am J Hum Genet 64:479-494

36. Roberts DL, Frerman FE, Kim J-JP 1996 Three-dimensional structure of human electron transfer flavoprotein to 2.1- $\AA$ resolution. Proc Natl Acad Sci U S A 93:14355-14260

37. Waters PJ, Parniak MA, Nowacki PN, Scriver CR 1998 In vitro expression analysis of mutations in phenylalanine hydroxylase: linking genotype to phenotype and structure to function. Hum Mutat 11:4-7
38. Andresen BS, Bross P, Udvari S, Kirk J, Gray G, Kmoch S, Chamoles N, Knudsen I, Winter V, Wilken B, Yokota I, Hart K, Packman S, Harpey JP, Saudubray JM, Hale DE, Bolund L, Kolvraa, N Gregersen 1997 The molecular basis of medium-chain acyl-CoA dehydrogenase (MCAD) deficiency in compound heterozygous patients: is there correlation between genotype and phenotype. Hum Mol Genet 6:695-707

39. Gregersen N, Winter VS, Corydon MJ, Corydon TJ, Rinaldo P, Ribes A, Martinez G, Bennett MJ, Vianey-Saban C, Bhala A, Hale DE, Lehnert W, Kmoch S, Roig M, Riudor E, Eiberg H, Andresen BS, Bross P, Bolund LA, Kolvraa S 1998 Identification of four new mutations in the short-chain acyl-CoA dehydrogenase (SCAD) gene in two patients: one of the variant alleles, $511 \mathrm{C} \Pi \mathrm{T}$, is present at an unexpectedly high frequency in the general population, as was the case for $625 \mathrm{G} \Pi \mathrm{A}$, together conferring susceptibility to ethylmalonic aciduria. Hum Mol Genet 7:619-627

40. Flott-Rahmel B, Falter C, Schluff P, Fingerhut R, Christensen E, Jakobs C, Musshoff U, Fautek JD, Deufel T, Ludolph A, Ullrich K 1997 Nerve cell lesions caused by 3-hydroxyglutaric acid: a possible mechanism for neurodegeneration in glutaric acidaemia type I. J Inherit Metab Dis 20:387-390

41. Ullrich K, Flott-Rahmel B, Schluff P, Musshoff, Das A, Lücke T, Steinfeld R, Christensen E, Jakobs C, Ludolph A, Neu A, Röper R 1999 Glutaric aciduria type I: pathomechanisms of neurodegeneration. J Inherit Metab Dis 22:392-403

42. Andresen BS, Bross P, Vianey-Saban C, Divry P, Zabot M, Roe CR, Nada MA, Byskov A, Kruse TA, Neve S, Kristiansen K, Knudsen I, Corydon MJ, Gregersen N 1996 Cloning and characterization of human very-long-chain acyl-CoA dehydrogenase cDNA, chromosomal assignment of the gene and identification in four patients of nine different mutations within the VLCAD gene. Hum Mol Genet 5:461-472

43. Shukla A, Dikshit M, Srimal RC 1995 Nitric oxide modulates blood-brain barrier permeability during infections with an inactivated bacterium. Neuroreport 6:16291632

44. Shinawi M, Gruener N, Lerner A 1998 CSF levels of carnitine in children with meningitis, neurologic disorders, acute gastroenteritis, and seizure. Neurology 50:1869-1871

45. Hathaway CA, Appleyard CB, Percy WH, Williams JL 1998 Collitis increases blood-brain barrier permeability in rabbits. FASEB J 12:A391

46. Land JM, Goulder P, Johnson A, Hockaday J 1992 Glutaric aciduria type I: an atypical presentation together with some observations upon treatment and the possible cause of cerebral damage. Neuropediatrics 23:322-326

47. Ribes A, Riudor E, Briones P, Christensen E, Campistol J, Millington DS 1992 Significance of bound glutarate in the diagnosis of glutaric aciduria type I. J Inherit Metab Dis 15:367-370 